\title{
Numerical Solution of Symmetric Positive Differential Equations
}

\section{By Theodore Katsanis}

\begin{abstract}
A finite-difference method for the solution of symmetric positive linear differential equations is developed. The method is applicable to any region with piecewise smooth boundaries. Methods for solution of the finite-difference equations are discussed. The finite-difference solutions are shown to converge at essentially the rate $O\left(h^{1 / 2}\right)$ as $h \rightarrow 0, h$ being the maximum distance between adjacent mesh-points.

An alternate finite-difference method is given with the advantage that the finite-difference equations can be solved iteratively. However, there are strong limitations on the mesh arrangements which can be used with this method.
\end{abstract}

Introduction. In the theory of partial differential equations there is a fundamental distinction between those of elliptic, hyperbolic and parabolic type. Generally each type of equation has different requirements as to the boundary or initial data which must be specified to assure existence and uniqueness of solutions, and to be well posed. These requirements are usually well known for an equation of any particular type. Further, many analytical and numerical techniques have been developed for solving the various types of partial differential equations, subject to the proper boundary conditions, including even many nonlinear cases. However, for equations of mixed type much less is known, and it is usually difficult to know even what the proper boundary conditions are.

As a step toward overcoming this problem Friedrichs [1] has developed a theory of symmetric positive linear differential equations independent of type. Chu [2] has shown that this theory can be used to derive finite-difference solutions in two-dimensions for rectangular regions, or more generally, by means of a transformation, for regions with four corners joined by smooth curves. In this paper a more general finite-difference method for the solution of symmetric positive equations is presented (based on [3]). The only restriction on the shape of the region is that the boundary be piecewise smooth. It is proven that the finite-difference solution converges to the solution of the differential equation at essentially the rate $O\left(h^{1 / 2}\right)$ as $h \rightarrow 0, h$ being the maximum distance between adjacent mesh-points for a two-dimensional region. Also weak convergence to weak solutions is shown.

An alternate finite-difference method is given for the two-dimensional case with the advantage that the finite-difference equation can be solved iteratively. However, there are strong limitations on the mesh arrangements which can be used with this method.

1. Symmetric Positive Linear Differential Equations. Let $\Omega$ be a bounded open set in the $m$-dimensional space of real numbers, $R^{m}$. The boundary of $\Omega$ will be

Received May 18, 1967. Revised May 8, 1968. 
denoted by $\partial \Omega$, and its closure by $\bar{\Omega}$. It is assumed that $\partial \Omega$ is piecewise smooth. A point in $R^{m}$ is denoted by $x=\left(x_{1}, x_{2}, \cdots, x_{m}\right)$ and an $r$-dimensional vector-valued function defined on $\Omega$ is given by $u=\left(u_{1}, u_{2}, \cdots, u_{r}\right)$. Also let $\alpha^{1}, \alpha^{2}, \cdots, \alpha^{m}$ and $G$ be given $r \times r$ matrix-valued functions and $f=\left(f_{1}, f_{2}, \cdots, f_{r}\right)$ a given $r$ dimensional vector-valued function, all defined on $\Omega$ (at least). It is assumed that the $\alpha^{i}$ are piecewise differentiable. For convenience, let $\alpha=\left(\alpha^{1}, \alpha^{2}, \cdots, \alpha^{m}\right)$, so that we can use expressions such as

$$
\nabla \cdot(\alpha u)=\sum_{i=1}^{m} \frac{\partial}{\partial x_{i}}\left(\alpha^{i} u\right) .
$$

With this notation we can write the identity

$$
\sum_{i=1}^{m} \frac{\partial}{\partial x_{i}}\left(\alpha^{i} u\right)=\sum_{i=1}^{m} \frac{\partial \alpha^{i}}{\partial x_{i}} u+\sum_{i=1}^{m} \alpha^{i} \frac{\partial u}{\partial x_{i}}
$$

simply as

$$
\nabla \cdot(\alpha u)=(\nabla \cdot \alpha) u+\alpha \cdot \nabla u .
$$

The definitions for symmetric positive operators and admissible or semiadmissible boundary conditions were introduced by Friedrichs [1].

Let $K$ be the first-order linear partial differential operator defined by

$$
K u=\alpha \cdot \nabla u+\nabla \cdot(\alpha u)+G u .
$$

$K$ is symmetric positive if each component, $\alpha^{i}$, of $\alpha$ is symmetric and the symmetric part, $\left(G+G^{*}\right) / 2$, of $G$ is positive definite on $\bar{\Omega}$.

For the purpose of giving suitable boundary conditions, a matrix, $\beta$, is defined (a.e.) on $\partial \Omega$ by

$$
\beta=n \cdot \alpha,
$$

where $n=\left(n_{1}, n_{2}, \cdots, n_{m}\right)$ is defined to be the outer normal on $\partial \Omega$.

The boundary condition $M u=0$ on $\partial \Omega$ is semiadmissible if $M=\mu-\beta$, where $\mu$ is any matrix with nonnegative definite symmetric part, $\left(\mu+\mu^{*}\right) / 2$. If in addition, $\mathfrak{N}(\mu-\beta) \oplus \mathfrak{K}(\mu+\beta)=R^{r}$ on the boundary, $\partial \Omega$, the boundary condition is termed admissible. $(\mathscr{N}(\mu-\beta)$ is the null space of the matrix $(\mu-\beta)$.)

The problem is to find a function $u$ which satisfies

$$
\begin{array}{ll}
K u=f & \text { on } \Omega, \\
M u=0 & \text { on } \partial \Omega,
\end{array}
$$

where $K$ is symmetric positive.

Many of the usual partial differential equations may be expressed in this symmetric positive form, with the standard boundary conditions also expressed as an admissible boundary condition. This includes equations of both hyperbolic and elliptic type. However, the greatest interest lies in the fact that the definitions are completely independent of type. An example of potentially great practical importance is the Tricomi equation which arises from the equations for transonic fluid flow. The Tricomi equation is of mixed type, i.e., it is hyperbolic in part of the region, elliptic in part, and is parabolic along the line between the two parts.

The significance of the semiadmissible boundary condition is that this insures 
the uniqueness of a classical solution to a symmetric positive equation. On the other hand, the stronger, admissible boundary condition is required for existence. The existence of a classical solution is generally difficult to prove for any particular case, and depends on properties at corners of the region.

Let $\mathcal{H C}$ be the Hilbert space of all square integrable $r$-dimensional vector-valued functions defined on $\Omega$. The inner product is given by

$$
(u, v)=\int_{\Omega} u \cdot v
$$

where

$$
u \cdot v=\sum_{i=1}^{r} u_{i} v_{i}
$$

and

$$
\|u\|^{2}=(u, u) \text {. }
$$

A boundary inner product is defined by

$$
(u, v)_{B}=\int_{\partial \Omega} u \cdot v
$$

with the corresponding norm

$$
\|u\|_{B^{2}}=(u, u)_{B} \text {. }
$$

The adjoint operators $K^{*}$ and $M^{*}$ are defined by

$$
\begin{aligned}
& K^{*} u=-\alpha \cdot \nabla u-\nabla \cdot(\alpha u)+G^{*} u, \\
& M^{*} u=\left(\mu^{*}+\beta\right) u .
\end{aligned}
$$

We will make use of the following lemmas by Friedrichs.

Lemma 1.1 (First Identity). If $K$ is symmetric positive, then

$$
(v, K u)+(v, M u)_{B}=\left(K^{*} v, u\right)+\left(M^{*} v, u\right)_{B} .
$$

Lrmma 1.2 (Second Identity). If $K$ is symmetric positive, then

$$
(u, K u)+(u, M u)_{B}=(u, G u)+(u, \mu u)_{B} .
$$

Lemma 1.3. Suppose $u$ is a solution to (1.5) where $M$ is semiadmissible. Let $\lambda_{G}$ be the smallest eigenvalue of $\left(G+G^{*}\right) / 2$ in $\bar{\Omega}$. Then

$$
\|u\| \leqq\left(1 / \lambda_{G}\right)\|f\| \text {. }
$$

Lemma 1.4. Let u satisfy Eq. (1.5) where $M$ is semiadmissible. Further, assume that $\left(\mu+\mu^{*}\right) / 2$ is positive definite on $\partial \Omega$ with smallest eigenvalue $\lambda_{\mu}$. Then

$$
\|u\|_{B} \leqq\left(1 /\left(\lambda_{G} \lambda_{\mu}\right)^{1 / 2}\right)\|f\| \text {. }
$$

Lemma 1.3 insures the uniqueness of a classical solution, and also that it is well posed in $L^{2}$ for homogeneous boundary conditions.

By widening the class of solutions to (1.5) to include weak solutions it is quite easy to prove existence of a solution to a symmetric positive equation under only semiadmissible boundary conditions. We will use Friedrichs' definition of weak 
solution. Let $V=C_{1}(\Omega) \cap\left\{v \mid M^{*} v=0\right.$ on $\left.\partial \Omega\right\}$. A function $u \in \mathfrak{H C}$ (defined above) is a weak solution of (1.5) if $f \in \mathcal{K C}$ and for all $v \in V$

$$
(v, f)=\left(K^{*} v, u\right) \text {. }
$$

It follows from the "first identity" (1.12) that a classical solution is also a weak solution.

Friedrichs [1] proved the existence of weak solutions if $M$ is semiadmissible. He also showed that, if, in addition, $M$ is admissible and the weak solution is continuously differentiable, then the weak solution must also be a classical solution.

2. Finite-Difference Solution of Symmetric Positive Differential Equations. First we will express $K$ in a form slightly different from (1.3), by the use of (1.2). We have

$$
K u=2 \nabla \cdot(\alpha u)-(\nabla \cdot \alpha) u+G u .
$$

Using the concept of vectors whose components are themselves matrices or vectors leads to somewhat simpler notation for the application of Green's theorem.

Lemma 2.1 (Green's Theorem). Let $g$ be a continuously differentiable mdimensional vector-valued function defined on $\Omega \subset R^{m}$, with vector components in either $R, R^{r}$ or $R^{r} \times R^{r}$. Then

$$
\int_{\Omega} \nabla \cdot g=\int_{\partial \Omega} g \cdot n
$$

This result follows directly from the definitions, using Green's theorem.

We now integrate the equation $K u=f$ over any region $P \subset \Omega$ using (2.1) and Green's theorem to obtain

$$
\int_{P} K u=2 \int_{\partial P} \beta u-\int_{P}(\nabla \cdot \alpha) u+\int_{P} G u=\int_{P} f .
$$

By a suitable approximation to (2.3) the desired finite-difference equations will be obtained.

Let $H$ be a set of $N$ mesh-points for $\Omega$. It is not required for the theory that the mesh-points all lie in $\Omega$. With each mesh-point $x_{j} \in H$ we identify a mesh-region $P_{j} \subset \Omega$ by

$$
P_{j}=\left\{x|| x-x_{j}|<| x-x_{k} \mid, \forall x_{k} \in H, k \neq j ; x \in \Omega\right\} .
$$

If $P_{j}$ is adjacent to $P_{k}$ we say that $x_{j}$ is connected to $x_{k}$ (corresponding to the fact that the directed graph of the resulting matrix will have a directed path in both directions between $j$ and $k$, see $\left[4\right.$, p. 16]). Let $l_{j, k}=\left|x_{j}-x_{k}\right|$, where $x_{j}$ is connected to $x_{k}$, and let $h=\max l_{j, k}$. Now define $A_{j}$ to be the "volume" of $P_{j}$ and $L_{j, k}$ to be the "area" of the $(r-1)$-dimensional "surface" between $P_{j}$ and $P_{k}$. We put $\Gamma_{j, k}=\bar{P}_{j} \cap \bar{P}_{k}$. Fig. 1 illustrates mesh-points and corresponding mesh-regions for two dimensions. This concept of mesh-regions is based on the suggestions of MacNeal [5]. We will always use the notation $\sum_{j}$ to indicate a sum over all points, $x_{j}$, in $H$, and $\sum_{k}$ to indicate a sum over points, $x_{k}$, which are connected to some one point, $x_{j}$. 
The desired finite-difference equation can now be obtained by a suitable approximation to Eq. (2.3). We use the symbol $\doteqdot$ to indicate the discrete approximation that will be used for each expression. First

$$
\int_{\Gamma_{j, k}} \beta u \doteqdot L_{j, k} \beta_{j, k} \frac{u_{j}+u_{k}}{2}
$$

where $u_{j}=u\left(x_{j}\right)$ and $\beta_{j, k}$ is the value of $\beta$ for $P_{j}$ at the center of $\Gamma_{j, k}$. (Note that $\beta_{j, k}=-\beta_{k, j}$.) The approximation to the next term of Eq. (2.3) requires approximating $u$ with $u_{j}$ first, and then applying Green's theorem before approximating $\alpha$. With this we obtain

$$
\int_{P j}(\nabla \cdot \alpha) u \doteqdot \int_{P j}(\nabla \cdot \alpha) u_{j}=\int_{\partial P j} \beta u_{j} .
$$

The final approximation is then

$$
\int_{\Gamma_{j, k}} \beta u_{j} \doteqdot L_{j, k} \beta_{j, k} u_{j} .
$$

Equations (2.4) and (2.6) take care of the integration over the interface between any $P_{j}$ and $P_{k}$. Now we need to make an approximation for the boundary sides. It will be convenient to be able to subdivide $\bar{P}_{j} \cap \partial \Omega$ into more than one piece. We will label each piece $\Gamma_{j, B}$ and we will use the convention that $\sum_{B}$ will mean a summation over the $B$ for just one $j$. We use $l_{j, B}$ to denote the distance from $x_{j}$ to $x_{B}$, where $x_{B}$ is located at the "center" of $\Gamma_{j, B}$ and $L_{j, B}$ is used for the "area" of $\Gamma_{j, B}$. Also $\beta_{j, B}=\beta\left(x_{B}\right)$. This notation is indicated for the two-dimensional case in Fig. 1. The desired approximations are now given by

$$
\begin{aligned}
& \int_{\Gamma_{j, B}} \beta u \doteqdot L_{j, B} \beta_{j, B} u_{B}, \\
& \int_{\Gamma_{j, B}} \beta u_{j} \doteqdot L_{j, B} \beta_{j, B} u_{j} .
\end{aligned}
$$

Finally the remaining terms in equation (2.3) are approximated by

$$
\begin{gathered}
\int_{P j} G u \doteqdot A_{j} G_{j} u_{j}, \\
\int_{P j} f \doteqdot A_{j} f_{j},
\end{gathered}
$$

where $G_{j}=G\left(x_{j}\right)$ and $f_{j}=f\left(x_{j}\right)$. Also we can approximate $\int K u$ by

$$
\int_{P j} K u \doteqdot A_{j}\left(K_{h} u\right)_{j}
$$

where $K_{h}$ is the finite-difference operator to be defined and which will approximate $K$. Using approximations (2.4) to (2.11) in Eq. (2.3) we arrive at the following definition of $K_{h}$,

$$
A_{j}\left(K_{h} u\right)_{j}=\sum_{k} L_{j, k} \beta_{j, k} u_{k}+\sum_{B} L_{j, B} \beta_{j, B}\left(2 u_{B}-u_{j}\right)+A_{j} G_{j} u_{j}
$$




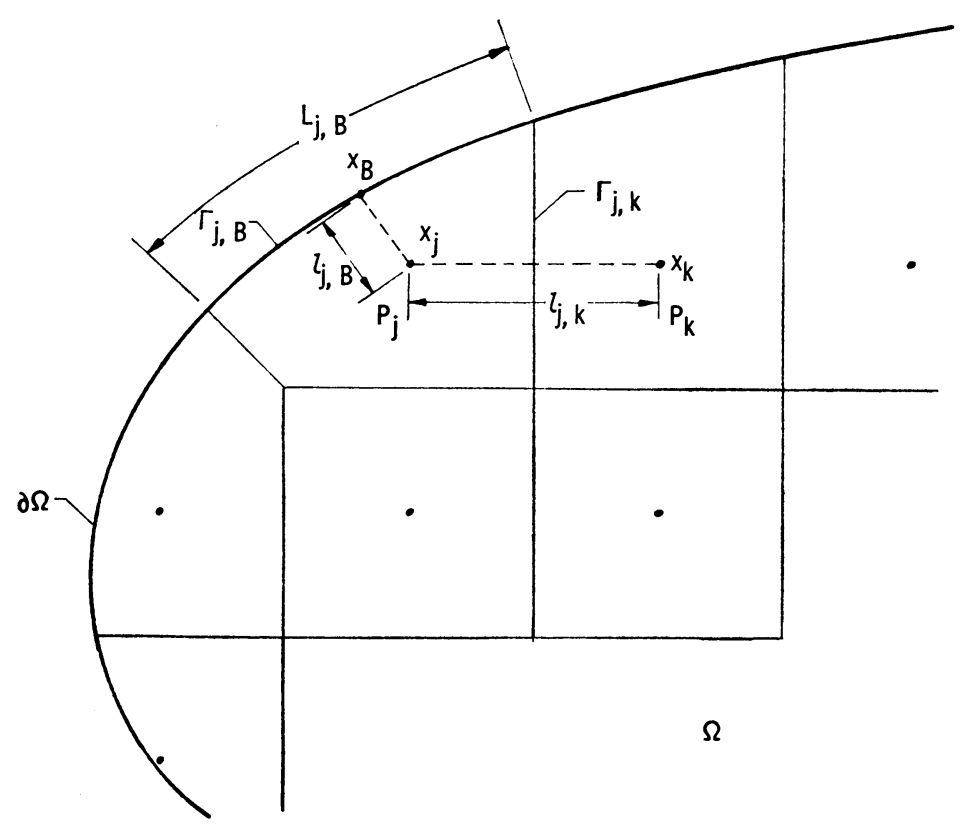

Figure 1. Typical mesh-regions in the two-dimensional case.

where $u$ here denotes a discrete function defined on $\bar{H}=H \cup\left\{x_{B}\right\}$, and $u_{j}=u\left(x_{j}\right)$. We will seek to find a function defined on $\bar{H}$ and satisfying $\left(K_{h} u\right)_{j}=f_{j}$ for every $x_{j} \in H$. Of course the solution is not yet uniquely determined since there are more unknowns than equations. The boundary condition $M u=0$ will furnish us with the necessary information to determine $u$ uniquely on $H$ (but not necessarily on all of $\bar{H}$ ).

Using $M_{h}$ to denote the boundary operator used to approximate $M$, we make the following definition

$$
\left(M_{h} u\right)_{j, B}=\mu_{j, B} u_{j}-\beta_{j, B}\left(2 u_{B}-u_{j}\right)
$$

for all $j$ where $P_{j}$ is a boundary polygon, and for all boundary surfaces of $P_{j}$ (each of which is associated with a point $x_{B}$ ). It is easily seen that $M_{h}$ is consistent with $M$ (i.e., $\left(M_{h} u\right)_{j, B} \rightarrow M u\left(x_{j, B}\right)$ as $h \rightarrow 0$ if $u$ is continuous). The reason for this choice of $M_{h}$ is that the condition $M_{h} u=0$ can be used to eliminate $u_{B}$ in $K_{h} u$ in a simple manner, and also we will be able to prove basic identities for the finitedifference operators analogous to those for the continuous operators (Eqs. (1.12) and (1.13)).

The existence and uniqueness of a solution to the finite-difference equation and the convergence to a continuous solution as $h \rightarrow 0$ depends on proving the basic identities for the discrete operators. Let $\mathfrak{F C}_{h}$ be the finite-dimensional Hilbert space of discrete functions defined on $H$. The inner product is given by

$$
(u, v)_{h}=\sum_{j} A_{j} u_{j} \cdot v_{j}
$$

and

$$
\|u\|_{h}^{2}=(u, u)_{h} .
$$


Also a "boundary" inner product is given by

$$
(u, v)_{B_{h}}=\sum_{j} \sum_{B} L_{j, B} u_{j, B} \cdot v_{j, B}
$$

for $P_{j}$ a boundary mesh region, and

$$
\|u\|_{B_{h}}^{2}=(u, u)_{B_{h}} .
$$

The discrete adjoint operators $K_{h}{ }^{*}$ and $M_{h}{ }^{*}$ are defined in the obvious way,

$$
\begin{gathered}
A_{j}\left(K_{h}^{*} u\right)_{j}=-\sum_{k} L_{j, k} \beta_{j, k} u_{k}-\sum_{B} L_{j, B} \beta_{j, B}\left(2 u_{B}-u_{j}\right)+A_{j} G_{j}^{*} u_{j}, \\
\left(M_{h}^{*} u\right)_{j, B}=u_{j, B}^{*} u_{j}+\beta_{j, B}\left(2 u_{B}-u_{j}\right) .
\end{gathered}
$$

We can now give the "first identity" for the discrete operators.

Lемма 2.2. If $K$ is symmetric positive, then

$$
\left(v, K_{h} u\right)_{h}+\left(v, M_{h} u\right)_{B_{h}}=\left(K_{h}^{*} v, u\right)_{h}+\left(M_{h}^{*} v, u\right)_{B_{h}}
$$

for any functions $u, v$ defined on $\bar{H}$.

Proof. Using the definitions, Eqs. (2.12) and (2.18), we have

$$
\begin{aligned}
\left(v, K_{h} u\right)_{h}-\left(K_{h}{ }^{*} v, u\right)_{h}=\sum_{j}[ & \sum_{k} L_{j, k} v_{j} \cdot \beta_{j, k} u_{k} \\
& +\sum_{B} L_{j, B} v_{j} \cdot \beta_{j, B}\left(2 u_{B}-u_{j}\right)+A_{j} v_{j} \cdot G_{j} u_{j} \\
& +\sum_{k} L_{j, k} \beta_{j, k} v_{k} \cdot u_{j} \\
& \left.+\sum_{B} L_{j, B} \beta_{j, B}\left(2 v_{B}-v_{j}\right) \cdot u_{j}-A_{j} G_{j}{ }^{*} v_{j} \cdot u_{j}\right] .
\end{aligned}
$$

By rearrangement, since $\beta_{j, k}=-\beta_{k, j}$, and since $\beta_{j, k}$ is symmetric we have

$$
\sum_{j} \sum_{k} L_{j, k} \beta_{j, k} v_{k} \cdot u_{j}=-\sum_{j} \sum_{k} L_{j, k} v_{j} \cdot \beta_{j, k} u_{k}
$$

and we see that all terms cancel with the exception of the boundary terms, so that

$$
\begin{aligned}
\left(v, K_{h} u\right)_{h}-\left(K_{h}^{*} v, u\right)_{h} & \\
& =\sum_{j} \sum_{B} L_{j, B}\left(v_{j} \cdot \beta_{j, B}\left(2 u_{B}-u_{j}\right)+\beta_{j_{,} B}\left(2 v_{B}-v_{j}\right) \cdot u_{j}\right) .
\end{aligned}
$$

On the other hand, using Eqs. (2.13) and (2.19),

$$
\begin{aligned}
\left(M_{h}^{*} v, u\right)_{B_{h}}-\left(v, M_{h} u\right)_{B_{h}}= & \sum_{j} \sum_{B} L_{j, B}\left(\mu_{j, B}^{*} v_{j} \cdot u_{j}+\beta_{j, B}\left(2 v_{B}-v_{j}\right) \cdot u_{j}\right) \\
& -\sum_{j} \sum_{B} L_{j, B}\left(v_{j} \cdot \mu_{j, B} u_{j}-v_{j} \cdot \beta_{j, B}\left(2 u_{B}-u_{j}\right)\right)
\end{aligned}
$$

which is the same as the right side of (2.21). Hence the "first identity" for the difference operators is proved.

The discrete operators have been defined so that $K_{h}+K_{h}{ }^{*}=G+G^{*}$ and $M_{h}+M_{h}{ }^{*}=\mu+\mu^{*}$. By letting $v=u$ in (2.20) we can prove the discrete "second identity" as for the continuous case.

Lemma 2.3. If $K$ is symmetric positive, then

$$
\left(u, K_{h} u\right)_{h}+\left(u, M_{h} u\right)_{B_{h}}=(u, G u)_{h}+(u, \mu u)_{B_{h}} .
$$


Using Eq. (2.13) and $M_{h} u=0$ we can eliminate $u_{B}$ from Eq. (2.12) so that the equation $K_{h} u=f$ can be reduced to

$$
\sum_{k} L_{j, k} \beta_{j, k} u_{k}+\sum_{\beta} L_{j, B} \mu_{j, B} u_{j}+A_{j} G_{j} u_{j}=A_{j} f_{j}, \quad \forall j .
$$

If we consider the case when $\Omega$ is two dimensional and rectangular, and the $P_{j}$ are all equal rectangles, we can compare (2.23) with the finite-difference equation obtained by Chu [2]. The equation obtained by $\mathrm{Chu}$ is the same as (2.23) for interior rectangles, but is different for boundary rectangles.

Let $A$ be the $r N \times r N$ matrix of coefficients of (2.23). Letting $\langle u, v\rangle=\sum_{j} u_{j} \cdot v_{j}$, the ordinary vector inner product, we have

$$
\langle u, A u\rangle=\left(u, K_{h} u\right)_{h}+\left(u, M_{h} u\right)_{B_{h}} .
$$

Hence, by the "second identity" (2.22), $A$ has positive definite symmetric part which shows that $A$ is nonsingular. We can also obtain an a priori bound for $\|u\|_{h}$ just as in the continuous case.

Lemma 2.4. Suppose $u$ is a solution to $K_{h} u=f, M_{h} u=0$, where $K$ is symmetric positive and $M$ is semiadmissible. Then

$$
\|u\|_{h} \leqq\left(1 / \lambda_{G}\right)\|f\|_{h} .
$$

If in addition, $\left(\mu+\mu^{*}\right)$ is positive definite on $\partial \Omega$, then

$$
\|u\|_{B_{h}} \leqq \frac{1}{\left(\lambda_{G} \lambda_{u}\right)^{1 / 2}}\|f\|_{h} .
$$

These bounds are obtained from the "second identity."

It is possible to show that the solution of the finite-difference equation (2.23) converges strongly to a continuously differentiable solution of equation (1.5), under the proper hypotheses. For simplicity we prove convergence only for the case when $\Omega$ is two dimensional $(m=2)$. Extension to regions in higher dimensions, with the same rate of convergence, follows directly. To allow the type of comparison we wish to make we will define operators mapping $\mathfrak{H}$ into $\mathfrak{H C}_{h}$ and vice versa. Let $r_{h}: \mathfrak{F C} \rightarrow \mathfrak{F C}_{h}$ be the projection defined by

$$
\left(r_{h} u\right)_{j}=u\left(x_{j}\right) \quad \text { for all } x_{j} \in H .
$$

In the other direction, let $p_{h}: \mathfrak{F}_{h} \rightarrow \mathfrak{H}$ be an injection mapping defined by

$$
p_{h} u_{h}(x)=\left(u_{h}\right)_{j}, \quad \text { for all } x \in P_{j} .
$$

We immediately have the following relations,

$$
\begin{aligned}
r_{h} p_{h} & =I, \\
\left\|p_{h} u_{h}\right\| & =\left\|u_{h}\right\|_{h} \quad \text { for all } u_{h} \in \mathfrak{F C}_{h} .
\end{aligned}
$$

We can now state our basic convergence theorem for two-dimensional regions.

'Theorem 2.1. Suppose that $u \in C^{2}(\bar{\Omega})$ satisfies

$$
\begin{array}{ll}
K u=f & \text { on } \Omega \subset R^{2}, \\
M u=0 & \text { on } \partial \Omega,
\end{array}
$$

where $K$ is symmetric positive, and $\mu+\mu^{*}$ is positive definite on $\partial \Omega$. For any given 
$h>0$, let $H_{h}$ be a set of associated mesh-points such that the maximum distance between connected nodes is less than $h$ and also that $L_{j, k}, L_{j, B}$ and $\left|x-x_{j}\right|$ for $x \in P_{j}$ are all less than $h . I t$ is assumed that the mesh is sufficiently regular so that $h^{2} / A_{j}$ for each $P_{j}$ is bounded independently of $h$ by a constant $K_{1}>0$, which is possible for sufficiently nice regions. Also it is assumed that a uniform rectangular mesh is used for all $P_{j}$ any point of which is at a distance greater than $K_{2}$ h from $\partial \Omega$, where $K_{2}$ is a positive constant. It is assumed that $\alpha \in C^{2}(\bar{\Omega})$.

Let $u_{h} \in \mathfrak{F}_{h}$ be the unique solution to

$$
K_{h} u_{h}=r_{h} f \text { on } H_{h}, \quad M_{h} u_{h}=0 .
$$

Then $\left\|p_{h} u_{h}-u\right\|=O\left(h^{\nu}\right)$ as $h \rightarrow 0$ for any positive $\nu<1 / 2$.

$\mathrm{Chu}$ [2] proved convergence of his finite-difference scheme, where $\Omega$ is a rectangle or a region with four corners, but the rate of convergence was not established.

Proof. Define $w_{h}=u_{h}-r_{h} u$. Let $\lambda_{G}$ be the smallest eigenvalue of $\left(G+G^{*}\right) / 2$ in $\bar{\Omega}$. Using the "second identity" (2.22), we have

$$
\left\|w_{h}\right\|_{h}^{2} \leqq\left(1 / \lambda_{G}\right)\left[\left(w_{h}, K_{h} w_{h}\right)_{h}+\left(w_{h}, M_{h} w_{h}\right)_{B_{h}}\right]
$$

Using the Cauchy-Schwartz inequality, we have

$$
\left\|w_{h}\right\|_{h}^{2} \leqq\left(1 / \lambda_{G}\right)\left(\left\|w_{h}\right\|_{h}\left\|K_{h} w_{h}\right\|_{h}+\left\|w_{h}\right\|_{B_{h}}\left\|M_{h} w_{h}\right\|_{B_{h}}\right)
$$

We will show that $\left\|K_{h} w_{h}\right\|_{h}=O\left(h^{1 / 2}\right)$ and $\left\|M_{h} w_{h}\right\|_{B_{h}}=O(h)$, as $h \rightarrow 0$. We shall need the following lemma.

Lemma 2.5. Let $g$ be a function defined on a finite region $P \subset R^{2}$, and suppose that $g$ satisfies a Lipschitz condition, i.e., there is a constant $K_{3}>0$ such that $|g(x)-g(y)| \leqq K_{3}|x-y|$, for all $x, y \in P$. Then, if $A_{0}$ is the area of $P$ and $\left|x-x_{0}\right| \leqq h$ in $P$,

$$
\left|g\left(x_{0}\right)-\frac{1}{A_{0}} \int_{P} g(x)\right| \leqq K_{3} h .
$$

We proceed now with the proof of the theorem. Let $\Omega_{1}$ denote that portion of $\Omega$ consisting of those $P_{j}$ which are rectangular, and let $\Omega_{2}$ denote the rest of the $P_{j}$. From the hypothesis we see that the area of $\Omega_{2}$ is less than the length of $\partial \Omega$ times $K_{2} h$. We have now that

$$
\left\|K_{h} w_{h}\right\|_{h}^{2}=\sum_{j \in J_{1}} \int_{P_{j}}\left(K u\left(x_{j}\right)-\left(K_{h} r_{h} u\right)_{j}\right)^{2}+\sum_{j \in J_{2}} \int_{P_{j}}\left(K u\left(x_{j}\right)-\left(K_{h} r_{h} u\right)_{j}\right)^{2},
$$

where

$$
J_{i}=\left\{j \mid P_{j} \subset \Omega_{i}\right\}, \quad i=1,2 .
$$

To simplify notation we will use $u_{j}$ for $u\left(x_{j}\right)$ and $u_{B}$ for $u\left(x_{B}\right)$. We now obtain a suitable bound for $\left|K u\left(x_{j}\right)-\left(K_{h} r_{h} u\right)_{j}\right|$

$$
\begin{aligned}
\left|K u\left(x_{j}\right)-\left(K_{h} r_{h} u\right)_{j}\right| \leqq & \left|2 \nabla \cdot(\alpha u)\left(x_{j}\right)-\sum_{k} \frac{L_{j, k}}{A_{j}} \beta_{j, k}\left(u_{j}+u_{k}\right)-2 \sum_{B} \frac{L_{j, B}}{A_{j}} \beta_{j, B} u_{B}\right| \\
& +\left|(\nabla \cdot \alpha) u\left(x_{j}\right)-\sum_{k} \frac{L_{j, k}}{A_{j}} \beta_{j, k} u_{j}-\sum_{B} \frac{L_{j, B}}{A_{j}} \beta_{j, B} u_{j}\right| .
\end{aligned}
$$

Consider the first term in the last expression above 


$$
\begin{aligned}
\mid 2 \nabla \cdot(\alpha u)\left(x_{j}\right)- & \sum_{k} \frac{L_{j, k}}{A_{j}} \beta_{j, k}\left(u_{j}+u_{k}\right)-2 \sum_{B} \frac{L_{j, B}}{A_{j}} \beta_{j, B} u_{B} \mid \\
\leqq & \left|2 \nabla \cdot(\alpha u)\left(x_{j}\right)-\frac{2}{A_{j}} \int_{P_{j}} \nabla \cdot(\alpha u)\right| \\
& +\frac{1}{A_{j}} \mid \sum_{k} \int_{\Gamma_{j, k}} 2\left(\beta u-(\beta u)_{j, k}\right) \\
& +\frac{1}{A_{j}}\left|\sum_{k} \int_{\Gamma_{j, k}} \beta_{j, k}\left(2 u_{j, k}-\left(u_{j}+u_{k}\right)\right)\right| .
\end{aligned}
$$

By Lemma 2.5, since $\alpha$ and $u \in C^{2}(\bar{\Omega})$ imply that their derivatives satisfy a Lipschitz condition,

$$
\left|2 \nabla \cdot(\alpha u)\left(x_{j}\right)-\frac{2}{A_{j}} \int_{P_{j}} \nabla \cdot(\alpha u)\right|=O(h) .
$$

We consider now the case when $j \in J_{1}$, so that $P_{j}$ is a rectangle with $x_{j}$ at the center.

Since $u \in C^{2}(\Omega)$, we have

$$
\begin{aligned}
& u_{j}=u_{j, k}-\frac{l_{j, k}}{2} u_{j, k}^{\prime}+\frac{l_{j, k}^{2}}{(4) 2} u^{\prime \prime}\left(\xi_{1}\right), \\
& u_{k}=u_{j, k}+\frac{l_{j, k}}{2} u_{j, k}^{\prime}+\frac{l_{j, k}^{2}}{(4) 2} u^{\prime \prime}\left(\xi_{2}\right),
\end{aligned}
$$

where the derivatives are directional derivatives in the direction $x_{k}-x_{j}$. Hence, if $\left|u^{\prime \prime}\right|<K_{3}$ in $\Omega$, we have

$$
\left|2 u_{j, k}-\left(u_{j}+u_{k}\right)\right|<\left(K_{3} / 4\right) h^{2}
$$

This means that

$$
\left|\int_{\Gamma_{j, k}} \beta_{j, k}\left(2 u_{j, k}-\left(u_{j}+u_{k}\right)\right)\right| \leqq L_{j, k}\left\|\beta_{j, k}\right\|\left|2 u_{j, k}-\left(u_{j}+u_{k}\right)\right|=O\left(h^{3}\right)
$$

when $j \in J_{1}$.

We now examine a Taylor series expansion for $\beta u$ about the point $x_{j, k}=$ $\left(x_{j}+x_{k}\right) / 2$.

$$
\begin{aligned}
& \beta\left(x_{j, k}+t z\right) u\left(x_{j, k}+t z\right)=(\beta u)_{j, k}+t\left(\frac{d}{d t}(\beta u)\right)_{j, k}+\frac{t^{2}}{2} g\left(\xi^{1}\right), \\
& \beta\left(x_{j, k}-t z\right) u\left(x_{j, k}-t z\right)=(\beta u)_{j, k}-t\left(\frac{d}{d t}(\beta u)\right)_{j, k}+\frac{t^{2}}{2} g\left(\xi^{2}\right)
\end{aligned}
$$

where $z$ is a unit vector orthogonal to $x_{j}-x_{k}, t$ is a scalar parameter, $g(\xi)=$ $\left(g_{1}\left(\xi_{1}\right), g_{2}\left(\xi_{2}\right), \cdots, g_{r}\left(\xi_{r}\right)\right), g_{i}$ is the $i$ th component of the vector $\left(d^{2} / d t^{2}\right)(\beta u)$, and $\xi_{i}$ is a point on the straight line between $x_{j, k}+\left(L_{j, k} / 2\right) z$ and $x_{j, k}-\left(L_{j, k} / 2\right) z$. Using (2.37) we obtain the following bound, 


$$
\left|\int_{\Gamma_{j, k}} \beta u-(\beta u)_{j, k}\right|=O\left(h^{3}\right) .
$$

Now, using (2.35), (2.36) and (2.38) in (2.34) we obtain

$$
\left|2 \nabla \cdot(\alpha u)\left(x_{j}\right)-\sum_{k} \frac{L_{j, k}}{A_{j}} \beta_{j, k}\left(u_{j}+u_{k}\right)\right|=O(h)
$$

for all $j \in J_{1}$, since $h^{2} / A_{j} \leqq K_{1}$ and the boundary terms are not present.

Consider now the second term on the right of (2.33):

$$
\begin{aligned}
& \left|(\nabla \cdot \alpha) u\left(x_{j}\right)-\sum_{k} \frac{L_{j, k}}{A_{j}} \beta_{j, k} u_{j}-\sum_{B} \frac{L_{j, B}}{A_{j}} \beta_{j, B} u_{j}\right| \\
& \leqq \\
& \quad\left|(\nabla \cdot \alpha) u\left(x_{j}\right)-\frac{1}{A_{j}} \int_{P_{j}}(\nabla \cdot \alpha) u\right|+\frac{1}{A_{j}}\left|\int_{P_{j}}(\nabla \cdot \alpha)\left(u-u_{j}\right)\right| \\
& \quad+\frac{1}{A_{j}}\left|\sum_{k} \int_{\Gamma_{j, k}}\left(\beta-\beta_{j, k}\right) u_{j}+\sum_{B} \int_{\Gamma_{j, B}}\left(\beta-\beta_{j, B}\right) u_{j}\right| \cdot
\end{aligned}
$$

By Lemma 2.5

$$
\left|(\nabla \cdot \alpha) u\left(x_{j}\right)-\frac{1}{A_{j}} \int_{P_{j}}(\nabla \cdot \alpha) u\right|=O(h) .
$$

Next, since $u$ satisfies a Lipschitz condition, $\left|x-x_{j}\right|<h$ for all $x \in P_{j}$, and since $\|\nabla \cdot \alpha\|$ is uniformly bounded in $\Omega$, we have

$$
\frac{1}{A_{j}}\left|\int_{P_{j}}(\nabla \cdot \alpha)\left(u-u_{j}\right)\right|=O(h) .
$$

Finally, since $\beta_{j, k}$ and $\beta_{j, B}$ are each evaluated at the midpoint of $\Gamma_{j, k}$ or $\Gamma_{j, B}$, respectively, we can use a Taylor series analysis, as in deriving equation (2.38), to obtain

$$
\frac{1}{A_{j}}\left|\sum_{k} \int_{\Gamma_{j, k}}\left(\beta-\beta_{j, k}\right) u_{j}+\sum_{B} \int_{\Gamma_{j, B}}\left(\beta-\beta_{j, B}\right) u_{j}\right|=O(h) .
$$

Combining (2.41), (2.42), and (2.43) in (2.40) we obtain

$$
\left|(\nabla \cdot \alpha) u\left(x_{j}\right)-\sum_{k} \frac{L_{j, k}}{A_{j}} \beta_{j, k} u_{j}-\sum_{B} \frac{L_{j, B}}{A_{j}} \beta_{j, B} u_{j}\right|=O(h) .
$$

Note that (2.44) holds for all $j$, not just for $j \in J_{1}$.

We can now substitute (2.39) and (2.44) in (2.33) to obtain

$$
\left|K u\left(x_{j}\right)-\left(K_{h} r_{h} u\right)_{j}\right|=O(h) \quad \text { for all } j \in J_{1} .
$$

We cannot obtain as good a bound for $\left|K u\left(x_{j}\right)-\left(K_{h} r_{h} u\right)_{j}\right|$ when $j \in J_{2}$, although (2.44) holds, since $\Gamma_{j, k}$ is not in general bisected by the line between $x_{j}$ and $x_{k}$. However, we can show that $\left|K u\left(x_{j}\right)-\left(K_{h} r_{h} u\right)_{j}\right|$ is uniformly bounded for $j \in J_{2}$, which is adequate since the area of $\Omega_{2}$ is of order $h$. The two inequalities which must be re-examined are (2.36) and (2.38). We now have, since $u$ and $(\beta u)$ satisfy Lipschitz conditions, that 


$$
\begin{gathered}
\left|\int_{\Gamma_{j, k}} \beta_{j, k}\left(2 u_{j, k}-\left(u_{j}+u_{k}\right)\right)\right|=O\left(h^{2}\right), \\
\left|\int_{\Gamma_{j, k}} \beta u-(\beta u)_{j, k}\right|=O\left(h^{2}\right), \\
\left|\int_{\Gamma_{j, B}} \beta u-(\beta u)_{j, B}\right|=O\left(h^{2}\right) .
\end{gathered}
$$

Using this, with the other results which still hold, we see that $\left|K u\left(x_{j}\right)-\left(K_{h} r_{h} u\right)_{j}\right|$ is uniformly bounded for $j \in J_{2}$, as $h \rightarrow 0$. Using this, together with (2.45) in (2.32) we obtain

$$
\left\|K_{h} w_{h}\right\|_{h}^{2}=O\left(h^{2}\right)+O(h)
$$

so that

$$
\left\|K_{h} w_{h}\right\|_{h}=O\left(h^{1 / 2}\right) .
$$

The next step is to show that $\left\|M_{h} w_{h}\right\|_{B_{h}}=O(h)$. We have

$$
\left\|M_{h} w_{h}\right\|_{B}=\left\|M_{h} r_{h} u\right\|_{B_{h}}
$$

since $M_{h} u_{h}=0$. Now

$$
\left\|M_{h} r_{h} u\right\|_{B_{h}}^{2}=\sum_{j} \sum_{B} L_{j, B}\left(\mu_{j, B} u_{j}-\beta_{j, B}\left(2 u_{B}-u_{j}\right)\right)^{2} .
$$

However, using the fact that $\beta_{j, B} u_{B}=\mu_{j, B} u_{B}$,

$$
\left|\mu_{j, B} u_{j}-\beta_{j, B}\left(2 u_{B}-u_{j}\right)\right|=O(h)
$$

since $u$ is differentiable, and $\|\mu\|$ and $\|\beta\|$ are uniformly bounded. This shows that

$$
\left\|M_{h} r_{h} u\right\|_{B_{h}}^{2}=O\left(h^{2}\right)
$$

since $\sum_{j, B} L_{j, B}$ is simply the length of $\partial \Omega$. This proves that

$$
\left\|M_{h} w_{h}\right\|_{B_{h}}=O(h) .
$$

Using (2.49) and (2.50) in (2.31), we see that

$$
\left\|w_{h}\right\|_{h^{2}}=\left\|w_{h}\right\|_{h} O\left(h^{1 / 2}\right)+\left\|w_{h}\right\|_{B_{h}} O(h) .
$$

From Lemma 2.4, $\left\|w_{h}\right\|_{B_{h}}$ must be bounded, since

$$
\begin{aligned}
\left\|w_{h}\right\|_{B_{h}} & \leqq\left\|u_{h}\right\|_{B_{h}}+\left\|r_{h} u\right\|_{B_{h}} \\
& \leqq \frac{1}{\left(\lambda_{G} \lambda_{\mu}\right)^{1 / 2}}\left\|r_{h} f\right\|_{h}+\left\|r_{h} u\right\|_{B_{h}}
\end{aligned}
$$

which is certainly uniformly bounded as $h \rightarrow 0$. Likewise $\left\|w_{h}\right\|_{h}$ is bounded. So from (2.51) we have

$$
\left\|w_{h}\right\|_{h}=O\left(h^{1 / 4}\right) .
$$

However, if we use (2.52) in (2.51) we get $\left\|w_{h}\right\|_{h}=O\left(h^{3 / 8}\right)$, or by repeating this procedure enough times, 


$$
\left\|w_{h}\right\|_{h}=O\left(h^{\nu}\right), \quad \text { for any positive } \nu<1 / 2 .
$$

Finally, we establish the convergence rate for $\left\|p_{h} u_{h}-u\right\|$. We have

$$
\left\|p_{h} u_{h}-u\right\| \leqq\left\|w_{h}\right\|_{h}+\left\|p_{h} r_{h} u-u\right\| .
$$

The last term can be estimated by

$$
\left\|p_{h} r_{h} u-u\right\|^{2}=\sum_{j} \int_{P_{j}}\left(u_{j}-u\right)^{2}=O\left(h^{2}\right) .
$$

Using (2.53) and (2.55) in (2.54) we get

$$
\left\|p_{h} u_{h}-u\right\|=O\left(h^{\nu}\right)+O(h)=O\left(h^{\nu}\right), \quad \text { for any positive } \nu<1 / 2 .
$$

This completes the proof of Theorem 2.1.

This finite-difference method can be applied to the Tricomi equation ([1], [3]). It is worthwhile noting that the solution obtained by the finite-difference solution of the symmetric positive form of the Tricomi equation consists of derivatives of the stream function, which corresponds to velocities in the physical problem. Hence, even though we have a convergence rate which is less than $O\left(h^{1 / 2}\right)$, it is essentially equivalent to a convergence rate of $O\left(h^{3 / 2}\right)$ if the original second-order equation were solved directly for the stream function.

If a rectangular mesh is used, we can partition the matrix $A$ so as to be block tridiagonal. The matrix equation can then be solved by the block tridiagonal algorithm ([6] and [4, p. 196]). Schecter [6] shows that this algorithm is valid for any matrix with definite symmetric part. We have already shown that $A$ has positive definite symmetric part. Schecter [6], also suggests an alternate procedure for reducing the computer storage requirements in solving the matrix equation.

An alternate method of solution may be possible in some cases. $A$ may be decomposed as $A=D+S$ where $D$ is Hermitian and $S$ is skew symmetric. If the smallest eigenvalue, $\lambda_{D}$, of $D$ is larger than the spectral radius, $\rho(S)$, of $S$, then $\left\|D^{-1} S\right\|<1$. In this case we can use a simple iterative method. Let $u^{(0)}$ be arbitrary, and define $u^{(i)}$ recursively by $D u^{(i)}=-S u^{(i-1)}+f$. In this case $\lim _{i \rightarrow \infty} u^{(i)}=u$. In general, though, the eigenvalues of $D$ will not be sufficiently large for this simple method to work. However, the original finite difference equations can be modified in some cases by the addition of a "viscosity" term, so as to obtain a convergent iterative procedure for the solution of the matrix equation. This will be discussed further in the next section.

We can consider the discrete analogue of a weak solution. Let $V_{h}$ be the set of discrete functions, $v_{h}$, defined on $\bar{H}$ and satisfying $M_{h}{ }^{*} v_{h}=0$. For a discrete weak solution, $u_{h}$, we would then require that

$$
\left(K_{h}^{*} v_{h}, u_{h}\right)_{h}=\left(v_{h}, r_{h} f\right)_{h} \quad \text { for all } v \in V_{h} .
$$

From the "first identity" (2.20) we have then

$$
\left(v_{h}, r_{h} f\right)_{h}=\left(v_{h}, K_{h} u_{h}\right)_{h}+\left(v_{h}, M_{h} u_{h}\right)_{B_{h}} \quad \text { for all } v \in V_{h} .
$$

We see from this that $\left(K_{h} u_{h}\right)_{j}=f_{j}$ for all $P_{j}$ which are not on the boundary, by choosing $\left(v_{h}\right)_{j}=1$, and $\left(v_{h}\right)_{k}=0$ for $k \neq j$. Because of the discrete nature of the equations we are not assured of $u_{h}$ satisfying the boundary conditions. However, 
conversely, if $u_{h}$ satisfies $K_{h} u_{h}=r_{h} f$ and $M_{h} u_{h}=0$ we see immediately that (2.57) must be satisfied.

Chu [2] has shown weak convergence of his finite-difference solution to a weak solution of a symmetric positive equation and Cea [7] has investigated generally the question of weak or strong convergence of approximate solutions to weak solutions of elliptic equations. Using these ideas, we can prove weak convergence of our finite-difference solutions to weak solutions of symmetric positive equations.

TheOREm 2.2. For any $h>0$, let $H_{h}$ be a set of mesh points satisfying the requirements of Theorem 2.1. It is assumed that $\alpha \in C^{2}(\bar{\Omega})$. Let $u_{h}$ be the unique solution to $K_{h} u_{h}=r_{h} f, M_{h} u_{h}=0$.

If $\left\{h_{i}\right\}_{i=1}^{\infty}$ is a positive sequence converging to zero, then $\left\{p_{h_{i}} u_{h_{i}}\right\}_{i=1}^{\infty}$ has a subsequence which converges weakly in $H$ to a weak solution, $u$, of $E q$. (1.5), that is $\left(K^{*} v, u\right)=(v, f)$ for all $v \in V$.

Furthermore, if $u$ is a unique weak solution, then $\left\{p_{h_{i}} u_{h_{i}}\right\}_{i=1}^{\infty}$ converges weakly to $u$.

Proof. First we note that $\left\|p_{h} u_{h}\right\|$ is bounded, since $\left\|p_{h} u_{h}\right\|=\left\|u_{h}\right\|_{h} \leqq\left(1 / \lambda_{G}\right)\left\|r_{h} f\right\|_{h}$, by Lemma 2.4. Hence, there is a subsequence of $\left\{p_{h_{i}} u_{h_{i}}\right\}$ that converges weakly to some $u \in \mathfrak{F C}$. (See Theorem 4.41-B, Taylor [8].) For convenience of notation we will suppress the subscripts on the $h$.

We have, for all $v \in V$,

$$
\begin{aligned}
\left|\left(K_{h}{ }^{*} r_{h} v, u_{h}\right)_{h}-\left(K^{*} v, p_{h} u_{h}\right)\right| & \\
& \leqq\left(\left\|K_{h}{ }^{*} r_{h} v-r_{h} K^{*} v\right\|_{h}+\left\|p_{h} r_{h} K^{*} v-K^{*} v\right\|\right)\left\|p_{h} u_{h}\right\| .
\end{aligned}
$$

But $\left\|p_{h} r_{h} K^{*} v-K^{*} v\right\| \rightarrow 0$, and in Theorem 2.1 we can substitute $K^{*}$ for $K$ in equation (2.49) to show that $\left\|K_{h}{ }^{*} r_{h} v-r_{h} K^{*} v\right\| \rightarrow 0$ (since $K_{h} w_{h}=r_{h} K u-K_{h} r_{h} u$ ). Since $\left\|p_{h} u_{h}\right\|$ is bounded,

$$
\lim _{h \rightarrow 0}\left|\left(K_{h}^{*} r_{h} v, u_{h}\right)_{h}-\left(K^{*} v, p_{h} u_{h}\right)\right|=0 .
$$

However, since $K^{*} v \in \mathfrak{K}$, we know that $\lim _{h \rightarrow 0}\left(K^{*} v, p_{h} u_{h}\right)=\left(K^{*} v, u\right)$.

We have shown, then, that

$$
\lim _{h \rightarrow 0}\left(K_{h}^{*} r_{h} v, u_{h}\right)_{h}=\left(K^{*} v, u\right) \quad \text { for all } v \in V .
$$

The discrete "first identity," Eq. (2.20), gives

$$
\left(K_{h}^{*} r_{h} v, u_{h}\right)_{h}+\left(M^{*} r_{h} v, u_{h}\right)_{B_{h}}=\left(r_{h} v, r_{h} f\right)_{h} .
$$

Hence

$$
\left|\left(K_{h}{ }^{*} r_{h} v, u_{h}\right)_{h}-\left(r_{h} v, r_{h} f\right)_{h}\right| \leqq\left\|M^{*} r_{h} v\right\|_{B_{h}}\left\|u_{h}\right\|_{B_{h}} .
$$

By Lemma 2.4 $\left\|u_{h}\right\|_{B_{h}} \leqq\left\|r_{h} f\right\|_{h} /\left(\lambda_{G} \lambda_{\mu}\right)^{1 / 2}$ which is bounded. Also, the proof of equation (2.50) shows that $\lim _{h \rightarrow 0}\left\|M^{*} r_{h} v\right\|_{B_{h}}=0$, for all $v \in V$, so that

$$
\lim _{h \rightarrow 0}\left|\left(K_{h}^{*} r_{h} v, u_{h}\right)_{h}-\left(r_{h} v, r_{h} f\right)_{h}\right|=0 .
$$

Further, it is obvious that

$$
\lim _{h \rightarrow 0}\left(r_{h} v, r_{h} f\right)_{h}=(v, f) .
$$


Combining (2.60), (2.63) and (2.64) gives

$$
\left(K^{*} v, u\right)=(v, f) \text { for all } v \in V,
$$

which completes the proof of the theorem.

3. Special Finite-Difference Scheme for Iterative Solution of Matrix Equation. As pointed out in Section 2, the matrix equation $A u=f$ can be solved by an iterative procedure if the eigenvalues of the diagonal coefficient matrix are sufficiently large compared to the eigenvalues of the off-diagonal coefficient matrix. Following the idea of Chu [2] we modify the finite-difference equation by adding a "viscosity" term which will have a diminishing effect on the finite-difference equations as $h \rightarrow 0$, and yet will assure the convergence of an iterative method. Unfortunately, the method is not applicable to every arrangement of mesh-points. In fact there are rather severe restrictions which must be met. The first requirement is that the difference in areas of adjacent mesh-regions be sufficiently small. This cannot be readily done along an irregular boundary, however, unless the boundary is modified. A problem arises if the boundary is modified. The boundary condition is given by $M u=(\mu-\beta) u=0$ on $\partial \Omega$. We need to extend $M$ to be defined in a neighborhood of the boundary. It is possible to extend $M$ continuously in a neighborhood of the boundary. However, if the direction of the boundary changes, $\beta$ changes drastically, and we have no assurance that $\mu$ will be positive definite. The second requirement then is that $M$ can be extended continuously over a neighborhood of the boundary, in such a way that $\mu$ will have positive definite symmetric part along the approximating boundary.

Let $\Omega_{h}$ be an approximation to $\Omega$. $\Omega_{h}$ will have to meet several requirements to be specified later. $H_{h}$ will denote a set of mesh-points associated with $\Omega_{h}$ and with maximum distance $h$ between connected nodes, and $\bar{H}_{h}$ will denote $H_{h} \cup\left\{x_{B}\right\}$. The discrete inner product is given by

$$
\left(u_{h}, v_{h}\right)=\sum_{j} A_{j}\left(u_{h}\right)_{j} \cdot\left(v_{h}\right)_{j}
$$

with the $A_{j}$ being the area of $P_{j} \subset \Omega_{h}$. Similarly, the "boundary" inner product. is changed so that the lengths, $L_{j, B}$, are the lengths along $\partial \Omega_{h}$.

We define now two new finite-difference operators, $\bar{K}_{h}$ and $\bar{M}_{h}$, by

$$
\begin{aligned}
\left(\bar{K}_{h} u\right)_{j} & =\left(K_{h} u\right)_{j}+\sum_{k} \sigma \frac{u_{j}-u_{k}}{l_{j, k}}+\sum_{B} \sigma \frac{u_{j}-u_{B}}{l_{j, B}}, \\
\left(\bar{M}_{h} u\right)_{j, B} & =\left(M_{h} u\right)_{j, B}-\frac{\sigma A_{j}}{L_{j, B} l_{j, B}}\left(u_{j}-u_{B}\right),
\end{aligned}
$$

where $\sigma$ is a positive number which must satisfy requirements to be specified later.

It will be useful to prove a slightly different version of the "second identity."

I.EмMа 3.1. If $K$ is symmetric positive, then

$$
\left(u_{h}, \bar{K}_{h} u_{h}\right)_{h}+\left(u_{h}, \bar{M}_{h} u_{h}\right)_{B_{h}}=\left(u_{h}, G u_{h}\right)_{h}+\left(u_{h}, \mu u_{h}\right)_{B_{h}}+\sum_{(j, k)} \frac{\sigma A_{j}}{l_{j, k}}\left(u_{j}-u_{k}\right)^{2:}
$$

where $\sum_{(j, k)}$ indicates a sum over every $(j, k)$ pair where $x_{j}$ is connected to $x_{k}$.

Proof. Using the "second identity" for $K_{h}$ and $M_{h}$, Eq. (2.22), we have 


$$
\begin{aligned}
\left(u_{h}, \bar{K}_{h} u_{h}\right)_{h}+\left(u_{h}, \bar{M}_{h} u_{h}\right)_{B_{h}}= & \left(u_{h}, G u_{h}\right)_{h}+\left(u_{h}, \mu u_{h}\right)_{B h} \\
& +\sum_{j} \sum_{k} \frac{\sigma A_{j}}{l_{j, k}} u_{j} \cdot\left(u_{j}-u_{k}\right) \\
& +\sum_{j} \sum_{B} \frac{\sigma A_{j}}{l_{j, B}} u_{j} \cdot\left(u_{j}-u_{B}\right) \\
& -\sum_{j} \sum_{B} \frac{\sigma A_{j}}{l_{j, B}} u_{j} \cdot\left(u_{j}-u_{B}\right) .
\end{aligned}
$$

The last two terms cancel. For the other term we have

$$
\sum_{j} \sum_{k} \frac{\sigma A_{j}}{l_{j, k}} u_{j} \cdot\left(u_{j}-u_{k}\right)=\sum_{(j, k)} \frac{\sigma A_{j}}{l_{j, k}}\left(u_{j}-u_{k}\right)^{2}
$$

which completes the proof.

Lemma 3.1 immediately assures the existence and uniqueness of a solution for the special finite-difference scheme. Using $\bar{M}_{h} u_{h}=0$ to eliminate $u_{B}$ from $\bar{K}_{h} u_{h}=$ $r_{h} f$, we obtain

$$
\sum_{k}\left(L_{j, k} \beta_{j, k}-\frac{\sigma A_{j}}{l_{j, k}} I\right) u_{k}+\left(A_{j} G_{j}+\sum_{k} \frac{\sigma A_{j}}{l_{j, k}} I+\sum_{B} L_{j, B} \mu_{j, B}\right) u_{j}=A_{j} f_{j}
$$

for all $x_{j} \in H_{h}$.

Let $A$ be the matrix of coefficients of (3.5).

LemMa 3.2. If $K$ is symmetric positive, then $\bar{K}_{h} u_{h}=r_{h} f, \bar{M}_{h} u_{h}=0$ has a unique solution on $H_{h}$.

Proof. The hypothesis implies that

$$
\langle u, A u\rangle=\left(u_{h}, \bar{K}_{h} u_{h}\right)_{h}+\left(u_{h}, \bar{M}_{h} u_{h}\right)_{B_{h}} .
$$

By Lemma 3.1 $A$ has positive definite symmetric part, and hence is nonsingular. Thus (3.5) defines $u_{h}$ uniquely on $H_{h}$.

Also it will be noted that the "second identity" of Lemma 3.1 will give the same a priori bounds for $\left\|u_{h}\right\|_{h}$ and $\left\|u_{h}\right\|_{B_{h}}$ as given by (2.25) and (2.26).

We will now show that the special finite-difference scheme converges to a smooth solution, under a number of hypotheses given in the theorem. The theorem also includes all the hypotheses needed to assure convergence of the iterative matrix solution. Though quite a number of requirements are given, there are only two essential restrictions, namely, that the areas $A_{j}$ must be nearly uniform, and that $M$ can be specified on a modified boundary in such a way that $\mu$ remains positive definite.

Theorem 3.1. Suppose that $u \in C^{2}(\bar{\Omega})$ satisfies $K u=f$ on $\Omega, M u=0$ on $\partial \Omega$, where $K$ is symmetric positive. For any $h>0$, let $\Omega_{h}$ be an approximation to $\Omega$, and let $H_{h}$ be a corresponding set of mesh points with maximum distance $h$ between connected nodes, and also with $L_{j, k}, L_{j, B}$, and $\left|x-x_{j}\right|$ for $x \in P_{j}$ all less than $h$. It is assumed that the following hypotheses are satisfied:

(i) There exists $K_{1}>0$, independent of $h$, such that for every $P_{j}$ we have $h^{2} / A_{j}<K_{1}$.

(ii) There exists $K_{2}>0$, independent of $h$, such that all $P_{j}$ with any point at $a$ distance greater than $K_{2} h$ from $\partial \Omega$ are equal rectangles.

(iii) There exists $K_{3}>0$, independent of $h$, such that for all $x \in \partial \Omega_{h}$, the distance from $x$ to $\partial \Omega$ is less than $K_{3} h$. 
(iv) There exists $K_{4}>0$, such that $M$ can be extended so as to satisfy a uniform Lipschitz condition at all points at a distance less than $K_{4}$ from $\partial \Omega$.

(v) $\Omega_{h}$ is such that $\mu=M+\beta$ has positive definite symmetric part on $\partial \Omega_{h}$.

(vi) Let $W$ be the set of points that are a distance less than $K_{4}$ from $\partial \Omega$. Then $\alpha, G$, and $f$ are all extended to be defined on $\Omega \cup W$ with $\alpha \in C^{2}(\Omega \cup W)$ and $G$ positive definite on $\Omega \cup W$.

(vii) There exists $K_{5}>0$, independent of $h$, such that all points, $x_{j}$, associated with a boundary polygon, $P_{j}$, are in the polygon, and at a sufficient distance, $l_{j, B}$, from any boundary node, $x_{B}$, of $P_{j}$ so that $A_{j} \leqq K_{5} L_{j, B} l_{j, B}$.

(viii) Either $\Omega_{h} \subset \Omega$ or else $u$ can be extended so that $u \in C^{2}\left(\bar{\Omega}_{h}\right)$.

(ix) $\sigma>\eta K_{1} \rho_{B}+d$, where $d>0$ and $\rho_{B}$ is the supremum of the spectral radius of $n \cdot \alpha(x)$ for $x \in \Omega \cup W$, where $n$ is any unit vector and $\eta$ is the maximum number of nodes connected to any one node.

(x) $\left|A_{j} / A_{k}-1\right|<d \lambda_{G}\left(h^{\prime}\right)^{2} /\left(\eta^{2} \sigma^{2} h\right)$, for all connected nodes, $x_{j}$ and $x_{k}$, where $\lambda_{G}$ is the smallest eigenvalue of $G$ in $\bar{\Omega}_{h}$, and $h^{\prime}=\min \left(l_{j, k}\right)$.

(xi) The length of $\partial \Omega_{h}$ is uniformly bounded.

Let $u_{h}$ be the unique solution to $\bar{K}_{h} u_{h}=r_{h} f, \bar{M}_{h} u_{h}=0$; then

$$
\left\|u_{h}-r_{h} u\right\|=O\left(h^{\nu}\right) \quad \text { as } h \rightarrow 0, \quad \text { for any positive } \nu<1 / 2 \text {. }
$$

Proof. Letting $w_{h}=u_{h}-r_{h} u$, and using the "second identity," (3.4), we see that the inequality (2.31) is still valid for $\bar{K}_{h}$ and $\bar{M}_{h}$,

$$
\left\|w_{h}\right\|_{h}^{2} \leqq\left(1 / \lambda_{G}\right)\left(\left\|w_{h}\right\|_{h}\left\|\bar{K}_{h} w_{h}\right\|_{h}+\left\|w_{h}\right\|_{B_{h}}\left\|\bar{M}_{h} w_{h}\right\|_{B_{h}}\right) \text {. }
$$

We have

$$
\bar{K}_{h} w_{h}=r_{h} f-\bar{K}_{h} r_{h} u
$$

hence

$$
\left\|\bar{K}_{h} w_{h}\right\|_{h} \leqq\left\|r_{h} K u-K_{h} r_{h} u\right\|_{h}+\left\|K_{h} r_{h} u-\bar{K}_{h} r_{h} u\right\|_{h} .
$$

In checking the proof of Theorem 2.1 we see that $r_{h} K u-K_{h} r_{h} u$ is the same as $K_{h} w_{h}$ (Theorem 2.1), hence the bound of (2.49) holds for this term;

$$
\left\|r_{h} K u-K_{h} r_{h} u\right\|_{h}=O\left(h^{1 / 2}\right) .
$$

For the other term we have

$$
\left\|\left(\bar{K}_{h}-K_{h}\right) r_{h} u\right\|_{h}^{2}=\sum_{j} A_{j} \sigma^{2}\left(\sum_{k} \frac{u_{j}-u_{k}}{l_{j, k}}+\sum_{B} \frac{u_{j}-u_{B}}{l_{j, B}}\right)^{2} .
$$

Let $J_{1}$ denote the set of subscripts for those $P_{j}$ which are equal rectangles and let $J_{2}$ denote the rest of the subscripts. When $j \in J_{1}$ we have only the term $\sum_{k}\left(u_{j}-u_{k}\right) / l_{j, k}$ to consider. Because of the rectangular arrangement of points we can use a Taylor series analysis to show that

$$
\left|\sum_{k} \frac{u_{j}-u_{k}}{l_{j, k}}\right|=O(h)
$$

so that

$$
\sum_{j \in J_{1}} A_{j} \sigma^{2}\left(\sum_{k} \frac{u_{j}-u_{k}}{l_{j, k}}\right)^{2}=O\left(h^{2}\right)
$$


On the other hand, when $j \in J_{2}$ we cannot do as well. However, we note that both $\left(u_{j}-u_{k}\right) / l_{j, k}$ and $\left(u_{j}-u_{B}\right) / l_{j, k}$ are uniformly bounded since $u$ has a bounded derivative. Also, by hypothesis (ii), $\sum_{j \in j_{2}} A_{j}=O(h)$, so that

$$
\sum_{j \in J_{2}} A_{j} \sigma^{2}\left(\sum_{k} \frac{u_{j}-u_{k}}{l_{j, k}}+\sum_{B} \frac{u_{j}-u_{B}}{l_{j, B}}\right)^{2}=O(h) .
$$

It is assumed, of course, that the number of nodes connected to any one node is bounded as $h \rightarrow 0$.

Now, using (3.10) and (3.11) in (3.9) we have

$$
\left\|\left(\bar{K}_{h}-K_{h}\right) r_{h} u\right\|_{h}=O\left(h^{1 / 2}\right) .
$$

Taking this together with (3.8) in (3.7) finally

$$
\left\|\bar{K}_{h} w_{h}\right\|_{h}=O\left(h^{1 / 2}\right) \text {. }
$$

It is necessary now to obtain a bound for $\left\|\bar{M}_{h} w_{h}\right\|_{B_{h}}$. Since $\bar{M}_{h} w_{h}=-\bar{M}_{h} r_{h} u$, we have

$$
\left\|\bar{M}_{h} w_{h}\right\|_{B_{h}} \leqq\left\|M_{h} r_{h} u\right\|_{B_{h}}+\left\|\left(\bar{M}_{h}-M_{h}\right) r_{h} u\right\|_{B_{h}} .
$$

We have

$$
\left\|M_{h} r_{h} u\right\|_{B}^{2}=\sum_{j} \sum_{B} L_{j, B}\left(\mu_{j, B}-\beta_{j, B}\left(2 u_{B}-u_{j}\right)\right)^{2} .
$$

We can establish a bound, since

$$
\begin{aligned}
\left|\mu_{j, B}-\beta_{j, B}\left(2 u_{B}-u_{j}\right)\right| \leqq & \left|\mu_{j, B}\left(u_{j}-u_{B}\right)\right| \\
& +\left|\left(\mu_{j, B}-\beta_{j, B}\right) u_{B}\right|+\left|\beta_{j, B}\left(u_{j}-u_{B}\right)\right| .
\end{aligned}
$$

The first and last term on the right are of order $h$, since $u$ is differentiable and $\|\mu\|$ and $\|\beta\|$ are bounded. By hypothesis (iv) $M$ satisfies a Lipschitz condition, and so does $u$. Since the distance from $x_{B}$ to $\partial \Omega$ is less than $K_{3} h$ by (iii) and $M u=0$ on $\partial \Omega$, we see that $\left|\left(\mu_{j, B}-\beta_{j, B}\right) u_{B}\right|=O(h)$. Since, by (xi), $\sum_{j} \sum_{B} L_{j, B}$ is uniformly bounded, we have

$$
\left\|M_{h} r_{h} u\right\|_{B_{h}}=O(h) .
$$

Also, by using (vii)

$$
\left\|\left(\bar{M}_{h}-M_{h}\right) r_{h} u\right\|_{B}^{2} \leqq \sum_{j} \sum_{B} L_{j, B} K_{5}^{2} \sigma^{2}\left(u_{j}-u_{B}\right)^{2}=O\left(h^{2}\right) .
$$

This shows that

$$
\left\|\bar{M} w_{h}\right\|_{B_{h}}=O(h) .
$$

We check now to see that $\left\|w_{h}\right\|_{h}$ and $\left\|w_{h}\right\|_{B_{h}}$ are bounded. We have, using the a priori bound for $\left\|u_{h}\right\|_{h}$,

$$
\left\|w_{h}\right\|_{h} \leqq\left(1 / \lambda_{G}\right)\left\|r_{h} f\right\|_{h}+\left\|r_{h} u\right\|_{h}
$$

which must be bounded since $f$ and $u$ are. In the same manner, $\left\|w_{h}\right\|_{B h}$ must be bounded. Using this fact together with (3.13) and (3.17) in (3.6) we have

$$
\left\|w_{h}\right\|_{h}=O\left(h^{1 / 4}\right) \text {. }
$$


Using now (3.19) in (3.6) we get $\left\|w_{h}\right\|_{h}=O\left(h^{3 / 8}\right)$ and by repeating the process as many times as needed we get

$$
\left\|w_{h}\right\|_{h}=O\left(h^{\nu}\right) \quad \text { for any positive } \nu<1 / 2 .
$$

This completes the proof of Theorem 3.1.

For the iterative solution of the matrix equation $A u=f$ we will split $A$ into a block diagonal part $D$, and off diagonal part $B$. (We will suppress the subscript $h$ on the finite-difference solution $u_{h}$.) Thus, from (3.5), the $j$ th block of $D$ is an $r \times r$ matrix,

$$
D_{j}=A_{j} G_{j}+\sum_{k} \frac{\sigma A_{j}}{l_{j, k}} I+\sum_{B} L_{j, B} \mu_{j, B}
$$

and a typical block element of $B$ is

$$
B_{j, k}=L_{j, k} \beta_{j, k}-\frac{\sigma A_{j}}{l_{j, k}} I
$$

and $A=D+B$. The iterative method is given by

$$
u^{(i+1)}=-D^{-1} B u^{(i)}+D^{-1} f
$$

where $u^{(0)}$ is arbitrary. The hypotheses of Theorem 3.1 assure the convergence of $u^{(i)}$ to $u$.

Theorem 3.2. For any $h>0$, let $\Omega_{h}$ and $H_{h}$ satisfy the hypotheses of Theorem 3.1. Let $u^{(0)}$ be an arbitrary vector defined on $H_{h}$, and let $\left\{u^{(i)}\right\}_{i=0}^{\infty}$ be a sequence defined recursively by

$$
u^{(i+1)}=-D^{-1} B u^{(i)}+D^{-1} f .
$$

Then $\lim _{i \rightarrow \infty} u^{(i)}=u$, where $A u=f$.

Proof. By the contraction mapping theorem it is sufficient to show that $\left\|D^{-1} B\right\|<1$ for some matrix norm. Let $v$ be an arbitrary vector defined on $H_{h}$, and let $w=D^{-1} B v$. Since $D w=B v$, we have $\langle w, D w\rangle=\langle w, B v\rangle$, or

$$
\begin{gathered}
\sum_{j} w_{j} \cdot\left(A_{j} G_{j}+\sum_{k} \frac{\sigma A_{j}}{l_{j, k}} I+\sum_{B} L_{j, B} \mu_{j, B}\right) w_{j} \\
\leqq \\
\frac{1}{2} \sum_{j} \sum_{k} w_{j} \cdot\left(\frac{\sigma A_{j}}{l_{j, k}} I-L_{j, k} \beta_{j, k}\right) w_{j} \\
\quad+\frac{1}{2} \sum_{j} \sum_{k} v_{k} \cdot\left(\frac{\sigma A_{j}}{l_{j, k}} I-L_{j, k} \beta_{j, k}\right) v_{k} .
\end{gathered}
$$

This last inequality follows from the fact that

$$
\langle w, H v\rangle \leqq \frac{1}{2}\langle w, H w\rangle+\frac{1}{2}\langle v, H v\rangle
$$

for any positive definite Hermitian matrix. We see that $\left(\sigma A_{j}\right) /\left(l_{j, k}\right) I-L_{j, k} \beta_{j, k}$ is positive definite, since

$$
\sigma A_{j} / l_{j, k} \geqq L_{j, k} \rho\left(\beta_{j, k}\right)
$$

by (i) and (ix). By rearranging the terms of (3.21) so as to have all the $w$ terms on the left and all the $v$ terms to the right, we obtain 


$$
\begin{aligned}
\sum_{j} w_{j} \cdot\left(A_{j} G_{j}\right. & \left.+\sum_{B} L_{j, B} \mu_{j, B}\right) w_{j}+\frac{1}{2} \sum_{j} \sum_{k} w_{j} \cdot\left(\frac{\sigma A_{j}}{l_{j, k}} I+L_{j, k} \beta_{j, k}\right) w_{j} \\
& \leqq \frac{1}{2} \sum_{j} \sum_{k} v_{j} \cdot\left(\frac{\sigma A_{k}}{l_{j, k}} I+L_{j, k} \beta_{j, k}\right) v_{j} .
\end{aligned}
$$

The last expression was obtained by interchanging $j$ and $k$, since

$$
\beta_{j, k}=-\beta_{k, j} .
$$

We can write (3.23) in the following form.

$$
\begin{gathered}
\sum_{j} w_{j} \cdot\left(A_{j} G_{j}+\sum_{B} L_{j, B} \mu_{j, B}\right) w_{j}+\frac{1}{2} \sum_{j} \sum_{k} w_{j} \cdot\left(\frac{\sigma A_{j}}{l_{j, k}} I+L_{j, k} \beta_{j, k}\right) w_{j} \\
\leqq \frac{1}{2} \sum_{j} \sum_{k} v_{j} \cdot\left(\frac{\sigma A_{j}}{l_{j, k}} I+L_{j, k} \beta_{j, k}\right) v_{j}+\frac{1}{2} \sum_{j} \sum_{k} \frac{\sigma}{l_{j, k}}\left(A_{k}-A_{j}\right) v_{j}{ }^{2}
\end{gathered}
$$

or

$$
\langle w, X w\rangle+\langle w, Y w\rangle \leqq\langle v, Y v\rangle+\langle v, Z v\rangle
$$

where $X, Y$, and $Z$ are matrices defined by (3.24).

We have already shown that $Y$ is positive definite (using (3.22)); hence we can define a norm by

$$
\|v\|_{Y^{2}}=\langle v, Y v\rangle .
$$

We will show that $D^{-1} B$ is a strict contraction in the $Y$ norm. First we will need some inequalities. We have

$$
\langle w, X w\rangle>\lambda_{G}\|w\|_{h}{ }^{2}
$$

By (i) and (ix) we have

$$
\langle w, Y w\rangle \leqq\left(\eta \sigma / h^{\prime}\right)\|w\|_{h^{2}} .
$$

Also $\langle w, Y w\rangle$ can be bounded below by using (i) and (ix):

$$
\langle v, Y v\rangle \geqq(d / 2 h)\|v\|_{h^{2}} .
$$

Finally, we have

$$
\langle v, Z v\rangle \leqq \Lambda\left(\eta \sigma / 2 h^{\prime}\right)\|v\|_{h^{2}}
$$

where $\Lambda=\max \left|A_{k} / A_{j}-1\right|$, for all connected nodes, $x_{j}$ and $x_{k}$. From the definition (3.26), and using (3.27) and (3.28) we have

$$
\langle w, X w\rangle+\langle w, Y w\rangle>\left(1+\lambda_{G} h^{\prime} / \eta \sigma\right)\|w\|_{Y^{2}} .
$$

On the other hand from (3.29) and (3.30)

$$
\langle v, Y v\rangle+\langle v, Z v\rangle \leqq\left[1+\frac{\eta \sigma \Lambda}{d}\left(\frac{h}{h^{\prime}}\right)\right]\|v\|_{Y}{ }^{2} .
$$

Substituting (3.31) and (3.32) in (3.25) we have

$$
\|w\|_{Y}{ }^{2}<\left(\frac{1+\frac{\eta \sigma \Lambda}{d}\left(\frac{h}{h^{\prime}}\right)}{1+\lambda_{G} h^{\prime} / \eta \sigma}\right)\|v\|_{Y}{ }^{2} .
$$


Since $w=D^{-1} B v$, and $v$ is arbitrary, we see that $\left\|D^{-1} B\right\|_{Y}<1$ since

$$
\Lambda<\frac{d \lambda_{G} h^{\prime}}{\eta^{2} \sigma^{2}}\left(\frac{h^{\prime}}{h}\right)
$$

by hypothesis $(\mathrm{x})$. This completes the proof of Theorem 3.2.

Of course, if $\Omega_{h}$ can be selected so that all the $A_{j}$ are equal, then hypothesis (x) is satisfied, and

$$
\left\|D^{-1} B\right\|_{Y}<\frac{1}{\left(1+\left(\lambda_{G} h^{\prime} / \eta \sigma\right)\right)^{1 / 2}} .
$$

In the special case where all the $P_{j}$ are equal rectangles, $\eta=4$, so that

$$
\left\|D^{-1} B\right\|_{Y}<\frac{1}{\left(1+\left(\lambda_{G} h^{\prime} / 4 \sigma\right)\right)^{1 / 2}} .
$$

4. Concluding Remarks. The Tricomi equation can be expressed in symmetric positive form. In [3] a Tricomi equation with a known analytical solution was solved numerically as an illustration of the numerical results which can be obtained. There was strong convergence to the analytical solution, but pointwise divergence. However, smoothing of the solution produced satisfactory numerical results.

5. Acknowledgement. I would like to express my appreciation to Professor Milton Lees for his guidance in this work.

National Aeronautics and Space Administration

Lewis Research Center

Cleveland, Ohio 44135

1. K. O. Friedrichs, "Symmetric positive linear differential equations," Comm. Pure Appl. Math., v. 11, 1958, pp. 333-418. MR $20 \# 7147$.

2. C. K. ChU, Type-Insensitive Finite Difference Schemes, Ph.D. Thesis, New York University, 1958.

3. T. Katsanis, Numerical Techniques for the Solution of Symmetric Positive Linear Differential Equations, Ph.D. Thesis, Case Institute of Technology, 1967.

4. R. S. VARgA, Matrix Iterative Analysis, Prentice-Hall, Princeton, N. J., 1962. MR 28 $\# 1725$.

5. R. II. MaCNeAL, "An asymmetrical finite difference network," Quart. Appl. Math., v. 11, 1953, pp. 295-310. MR $15,257$.

6. S. SCHECTER, "Quasi-tridiagonal matrices and type-insensitive difference equations," Quart. Appl. Math., v. 18, 1960/61, pp. 285-295. MR $22 \# 5133$.

7. J. Ć́. ", "Approximation variationelle des problèmes aux limites," Ann. Inst. Fourier (Grenoble), v. 14, 1964, fasc. 2, pp. 345-444. MR $30 \# 5037$.

8. A. E. TAYLOR, Introduction to Functional Analysis, Chapman \& Hall, London; Wiley, New York, 1958. MR $20 \# 5411$. 